\title{
Face-to-face single patch: A new technique to repair the commissures of the mitral valve
}

\author{
Samer Kassem, MD, PhD, ${ }^{a}$ Khalid Al-Faraidy, MD, ${ }^{\mathrm{b}}$ Yasser Elkady, MD, ${ }^{\mathrm{b}}$ and Ahmad Takriti, MD, ${ }^{\mathrm{c}}$ \\ Milan, Italy, Dhahran, Kingdom of Saudi Arabia, and Damascus, Syria
}

Mitral valve reconstruction is the procedure of choice for mitral regurgitation and has demonstrated excellent early and late results. ${ }^{1}$ Mitral valve repair in rheumatic valve disease is associated with durable long-term results and a lower rate of thromboembolic events compared with prosthetic mitral valve replacement. ${ }^{2}$

Some mitral valve pathologic entities are considered difficult to repair, including severe calcified commissures secondary to the rheumatic process, commissures destroyed by endocarditis, and commissural widespread prolapse late in the degenerative disease. Currently, only a few surgical techniques are available to repair mitral commissures. Autograft of the posterior tricuspid leaflet to the commissural mitral valve position, ${ }^{3}$ open commissurotomy in moderately fibrosed and calcified rheumatic mitral valve commissures, ${ }^{4}$ and commissural repair in mitral endocarditis, ${ }^{5}$ have all been well described but are associated with technical challenges and limitations.

Our objective was to propose a new technique for repairing the mitral commissure using an autologous pericardial patch, conserving the physiologic function of the valvular area. A single patch is implanted over the commissural zone, which is then divided into the anterior and posterior parts by a new artificial chord extended between the corresponding papillary muscle and trigone portion of the mitral prosthetic ring.

\section{SURGICAL TECHNIQUE}

In the present report we describe the repair of the posterior commissure of a rheumatic mitral valve using our newly developed technique. First, transesophageal echocardiography is performed to assess valvular function. Next, a strip of pericardium is prepared free from adipose and extrapleural tissue. Thereafter, both aortic and bicaval cannulations are achieved. Antegrade and retrograde cold blood

\footnotetext{
From the Centro Cardiologico Monzino IRCCS, ${ }^{\mathrm{a}}$ Unit II, Milan, Italy; Cardiac Center of the King Fahad Military Medical Complex, ${ }^{b}$ Dhahran, Kingdom of Saudi Arabia; and Damascus University Cardiovascular Surgical Hospital, ${ }^{\mathrm{c}}$ Damascus, Syria. Disclosures: Authors have nothing to disclose with regard to commercial support. Received for publication April 9, 2012; revisions received June 13, 2012; accepted for publication July 30, 2012; available ahead of print Aug 30, 2012.

Address for reprints: Samer Kassem, MD, PhD, Department of Cardiovascular Surgery, Centro Cardilogico Monziono IRCCS, Unit-II, Via Parea, no. 4, Milan 20138, Italy (E-mail: samer.kassem@yahoo.it).

J Thorac Cardiovasc Surg 2012;144:1523-7

$0022-5223 / \$ 36.00$

Copyright (C) 2012 by The American Association for Thoracic Surgery

http://dx.doi.org/10.1016/j.jtcvs.2012.07.079
}

cardioplegia, carbon dioxide insufflation of the operative field, and moderate hypothermia $\left(32^{\circ} \mathrm{C}\right)$ are routinely used. Inspection of the mitral valve is performed as usual, and the mechanisms of mitral stenosis and/or regurgitation are identified. The leaflets are accurately analyzed by close inspection to determine whether the anterior leaflet is pliable and the posterior is not extensively calcified. Then, if possible, the heads of the posterior papillary muscle are identified and inspected. The significant commissural fusion and calcification are carefully evaluated, and the extension of A3 and P3 fibrosis is identified. The nearest relatively healthy chords of the anterior and posterior leaflets to the bloc of commissure are demarcated (Figure 1, $A$ ). Thereafter, the $\mathrm{A} 3$ and $\mathrm{P} 3$ are detached along the mitral annulus, and the incision is extended to the free edges of the anterior and posterior leaflets, conserving the already distinguished native chords. Conservation of the mitral annulus in the trigone zone is essential for this technique, using it as a point of reference to measure the new chord. In addition, repair of the annulus in this zone is complicated and makes this technique very complex. The commissural calcifications and the fused chords are resected en bloc (Figure 1, $B$ ). The retracted secondary chordae of the anterior and posterior leaflets, in addition to the incision of the leaflets, are also excised. The morphology of the papillary muscle and location of its components with respect to the commissure are considered. A double-armed 4-0 polytetrafluoroethylene suture (Gore-Tex; Gore Medical, Flagstaff, Ariz) supported by a felt pledget is passed twice through the tip of the intermediate head of the posterior papillary muscle, using a backhand technique in the direction of the commissure, and fixed with multiple knots kept relatively loose to avoid the development of necrosis. The distance between the intermediate head of the posterior papillary muscle and corresponding trigone is used as a guide to determine the length of the new chord. Next, the assistant surgeon stretches the annulus adjacent to the trigone by a nerve hook, and a metal clip is placed across the 2 lengths of the new chord on the atrial face of the leaflet at the level of the trigone. A nonsliding knot is tied against the metal clip, which is subsequently removed (Figure 1, C).

A close analysis of the 3-dimensional defect will reveal 5 distinct components (Figure 1,B): the anterior leaflet (B-C), the posterior leaflet (A-D), 2 sets of the native chords (E-C and F-D), and the mitral annulus (A-B). The assistant stretches the components, and the surgeon measures them 

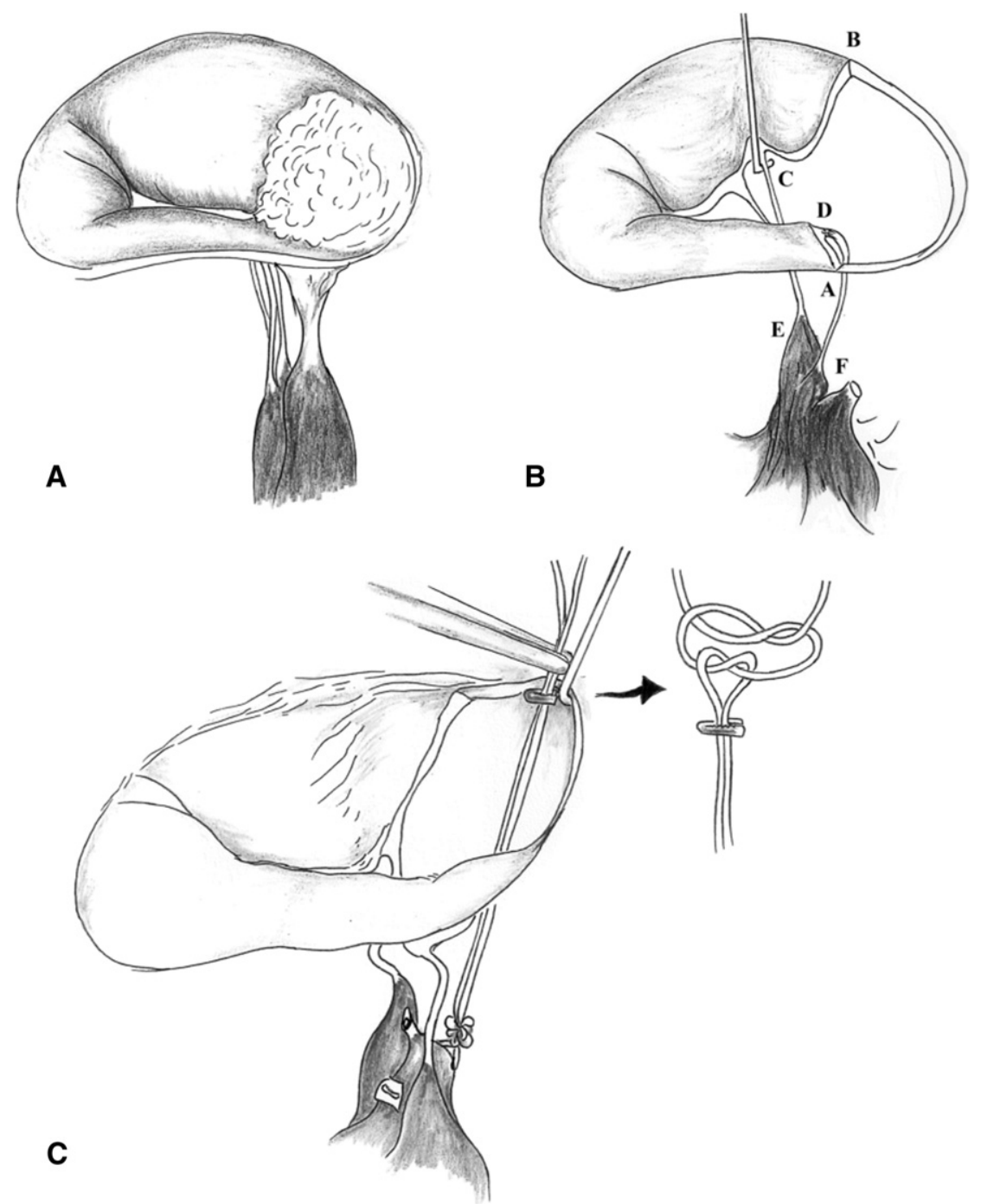

FIGURE 1. A, Illustration of calcification and fusion of posterior commissure, extension of A3 and P3 fibrosis, and attached and relatively healthy chords. $\mathrm{B}$, The commissural calcifications and fused chords are resected en bloc. The nearest relatively healthy chords of anterior and posterior leaflets to bloc of commissure are conserved. The illustration shows the 5 distinct components of the 3-dimensional defect: anterior leaflet (B-C), posterior leaflet (A-D), 2 sets of native chords (E-C and F-D), and mitral annulus (A-B). C, Illustration showing new chord anchored on medial head of the posterior papillary muscle. The assistant surgeon stretches the annulus adjacent to the right trigone using a nerve hook. Next, the surgeon places a metal clip across the 2 arms of the new chord at the level of the annulus. Several nonsliding knots are tied against the metal clip, which is subsequently removed.

individually using a free surgical thread and metal clips. For the suture to be effective without reducing the surface of the patch, the length of each thread's component must exceed by $1 \mathrm{~cm}$ the actual length of the same measured segment (Figure 2, A). A patch of previously harvested fresh autologous pericardium is carefully fashioned into a right-angle trapezoid shape according to the previously recorded measurements of the several components of the defect, taking into consideration that the smooth surface of the pericardium will ultimately be facing the left atrium. Therefore, the height of the trapezoid (a-b) will be sutured onto the annulus component (A-B), the long base (b-c-e) will face the anterior leaflet component, and the selected native anterior chord and short base (e-d-f) will face the posterior leaflet component and the selected native posterior chord (Figure 2, B). As shown in Figure 2, B, a triangle is resected from the trapezoid's surface between the 2 bases without passing the "c-d" line. The triangle gap of the patch will form the window filling of the neocommissure during diastole. Six stay sutures, of 5-0 yellow monofilament Radene (Dynek Pty Ltd, Hendon, Australia), are passed through the projected points (a, b, c, d, e, and f) of the patch, keeping it spread out (Figure 2,B). The patch is then lowered into position and fixed to the posterior and anterior angles of the annulus component ( $\mathrm{A}$ and $\mathrm{B}$ ) with the corresponding (a and b) stay sutures (Figure 2, C). The patch is attached 

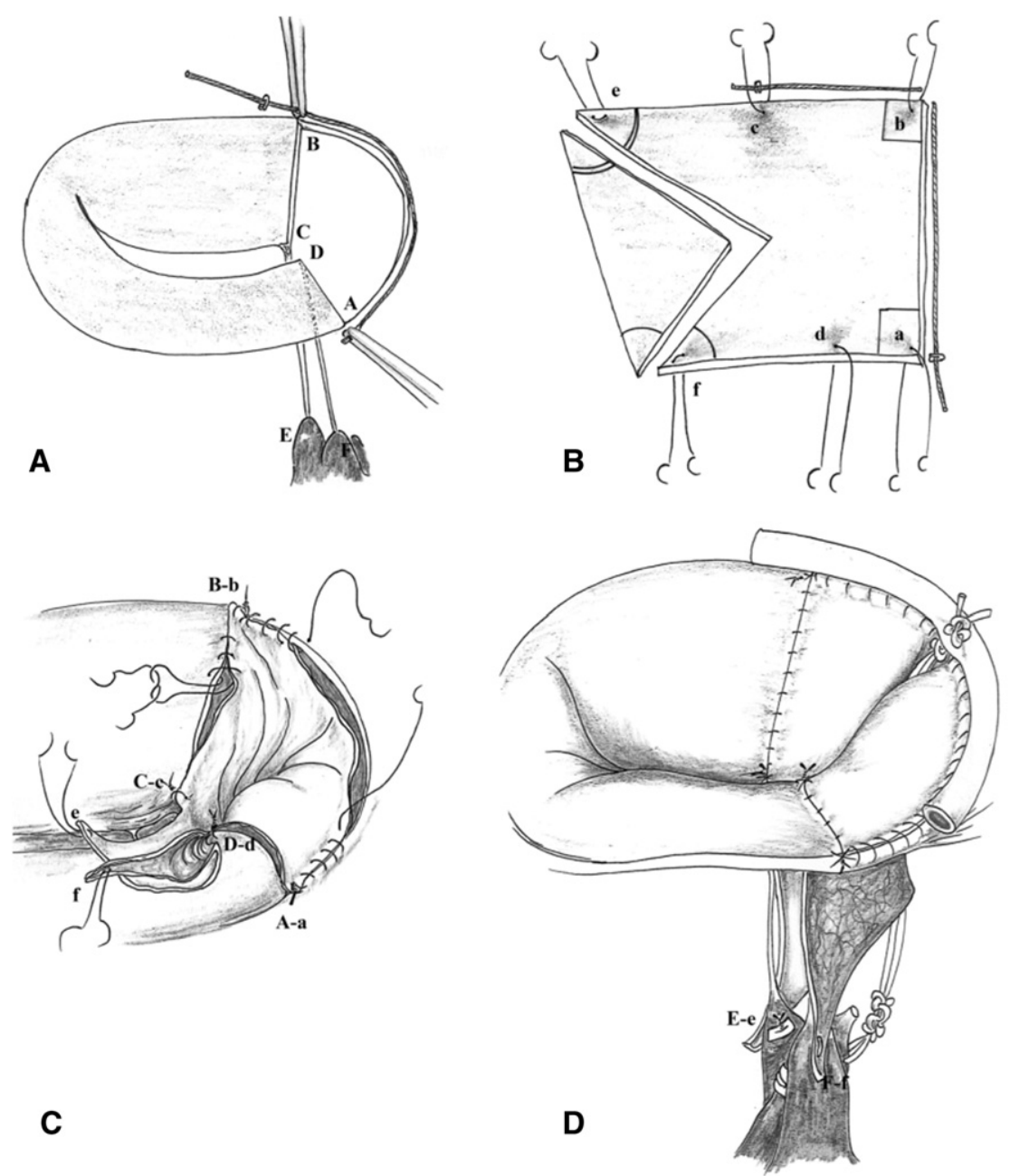

FIGURE 2. A, The 5 components are measured individually using a free surgical thread and metal clips. The length of each thread's component must exceed by $1 \mathrm{~cm}$ the actual length of the same measured segment. B, A patch of fresh autologous pericardium is fashioned into a right-angle trapezoid shape using the previously recorded measurements of the several components of the defect. A triangle is resected from the trapezoid's surface between the 2 bases without passing the c-d line. Six stay sutures, of 5-0 yellow monofilament Radene (Dynek), are passed through the projected points (a, b, c, d, e, and f) of the patch, keeping it spread out. C, The patch is fixed to the posterior and anterior angles of the annulus component (A and B) with corresponding (a and b) stay sutures and attached to the chordal inserts on the anterior and posterior leaflets $(C, D)$ with corresponding (c, d) stay sutures. Next, running 5-0 Radene suture is used to approximate the patch to the annulus component. The 2 windows between the patch and free margins of the resected anterior and posterior leaflets are closed with interrupted 5-0 Prolene sutures knotted toward the ventricular side of the suture lines. D, Illustration showing patch anchored to papillary head. A sized Physio annuloplasty ring is implanted, and Gore-Tex sutures are passed through corresponding trigone prosthetic sheath and tightly knotted.

to the chordal inserts on the anterior and posterior leaflets (C, D) by the corresponding (c, d) stay sutures (Figure 2, $C$ ). Next, a running 5-0 Radene suture is used to approximate the patch to the annulus component (Figure 2,C). The windows between the patch and free margins of the resected anterior and posterior leaflets are closed with interrupted 5-0 Prolene suture (Ethicon, Somerville, NJ) knotted toward the ventricular side of the suture lines (Figure 2, C). The length of the stretched anterior native chord (C-E) determines the length of the anterior patch papillary component (c-e), which is anchored to the anterior side of the anterior papillary head by the corresponding stay suture enforced by a small pledget (Figure 2,D). Similarly, the patch for the posterior papillary component (d-f) is measured and anchored to the posterior side of the posterior papillary head (Figure 2,D).

Finally, a Physio annuloplasty ring (Edwards Lifesciences, Irvine, Calif) is sized and added to complete the repair, ensuring that the untied Gore-Tex sutures (Gore Medical) inside the ring remain free. When the ring is placed in its final position, the Gore-Tex sutures (Gore Medical) are passed through the corresponding trigone prosthetic sheath 
and tightly knotted (Figure $2, B$ ). The valve is tested by injecting cold saline with considerable pressures to confirm that the neo-A 3 and neo-P3 are at the same level of the valvular plane and that the patch achieves the commissural shape by the stretched new chord under the filling pressure of the left ventricle.

This step-by-step description will perhaps make it easy to apply this technique to reconstruct the anterolateral commissure in the context of mitral valve repair, whenever needed.

\section{CLINICAL EXPERIENCE}

Recently, we have applied this technique for 4 selected patients with rheumatic mitral valve stenosis. Mitral valve stenosis was due to severe posteromedial commissural calcification in 3 patients and heavy rheumatic lesions of the anterolateral commissure in 1 patient. The annulus adjacent to the trigone was not extensively involved with the calcification process in any of these patients. Intraoperative transesophageal echocardiography and early follow-up transthoracic echocardiography showed the absence of mitral stenosis, no systolic anterior motion of the mitral valve, and no evidence of significant regurgitation. Because all these patients presented without atrial fibrillation, oral anticoagulant agents at a target international normalized ratio of 2.5 were used for only 3 months after mitral valve repair, in accordance with the European Society of Cardiology guidelines.

\section{DISCUSSION}

The particular anatomy of the commissures allows the mitral valve to splay open, providing a large orifice during diastole, and to close neatly during systole. Because the commissure lies between the annulus, adjacent to the trigone, anterior leaflet, posterior leaflet, and papillary muscle, it is anatomically situated in a complex 3-dimensional operable region.

The pericardium is an excellent material to repair the heart during management of certain acquired heart defects. ${ }^{6}$ Autologous pericardium is preferable, not only because it is free, but also because it is resistant and easy to handle, easily available, and nonimmunoreactive. It appears to be safe to use for repair of several types of congenital and acquired defects in the heart. In addition, patients will not refuse its use because of social, cultural, or religious reasons. Repair of rheumatic mitral valve insufficiency using autologous pericardium has been performed with excellent results. ${ }^{7,8}$

The new chord divides the atrial surface of the implanted patch into the anterior and posterior aspects. During systole, the ventricular pressures allow parts of both the atrial faces of the patch to bulge anteriorly and posteriorly, brushing against each other and creating good coaptation with wide surfaces. The distance E-F corresponds to the distance between the anterior surface of the anterior papillary head and the posterior face of the posterior papillary head. This distance corresponds to the thickness of the papillary muscle, when the papillary muscle is simple, single "type I." However, in other cases, it could be wider when the papillary muscle is divided, such as in type III and IV. ${ }^{9}$ We consider the e-f distance as the second side of a trapezium, and its length is determined by the ends of the 2 bases. The distance E-F is smaller than the side e-f distance, such that implantation of the patch creates 2 surfaces facing each other. Triangular resection of the trapezoid patch during preparation provides an open orifice of the commissural zone during diastole. The new chord is located between the head of the papillary muscle and the annulus adjacent to the trigone. The tip papillary-annulus distance is relatively fixed during cardiac diastole and systole; thus, movement of the new chord should be limited. The upper part of the new commissure (toward the trigone) is in contact with the new chord; thus, the trauma will be minimal. The lower part (toward the papillary) is distant from the new chord; however, we think the forces that secure coaptation between the anterior and posterior surfaces of the patch minimize the trauma during systole.

\section{CONCLUSIONS}

Our technique to repair the mitral commissure is simpler and can be indicated for patients who require extensive resection within the commissural area involving lateral scallops of both anterior and posterior leaflets. We prefer to use fresh autologous pericardium; however, we suggest the use of a bovine pericardial patch if the autologous pericardium is absent or unusable because of postoperative pericardial adhesions or previous pericarditis. Reconstruction of the mitral commissure with this technique, not only conserves the opening of the commissural area, but also helps regain the physiologic motion of the anterior mitral valve leaflet.

Although these early results in a small group of patients have been encouraging, meticulous long-term follow-up from a large series is critical to demonstrate the effectiveness and durability of this technique. Nevertheless, we believe that our face-to-face single pericardial patch technique will be a useful addition in the armamentarium of procedures currently available to repair the mitral valve.

\section{References}

1. Cohn LH, Couper GS, Arankl SE, Rizzo RJ, Kinchla NM, Collins JJ Jr. Long-term results of mitral reconstruction for regurgitation of the myxomatous mitral valve. J Thorac Cardiovasc Surg. 1994;107:143-50.

2. Chauvaud S, Fuzellier JF, Berrebi A, Deloche A, Fabiani JN, Carpentier A. Longterm (29 years) results of reconstructive surgery in rheumatic mitral valve insufficiency. Circulation. 2001;104(Suppl 1):I12-5.

3. Hvass U, Chatel D, Caliani J, Oroudji M. Mitral valve repairs using the posterior tricuspid leaflet and chordae: technique and results. Eur J Cardiothorac Surg. 1996;10:874-8.

4. Antunes MJ, Vieira H, Ferrão de Oliveira J. Open mitral commissurotomy: the "golden standard." J Heart Valve Dis. 2000;9:472-7.

5. Ushijima T, Kikuchi Y, Takata M, Yamamoto Y, Kawachi K, Watanabe G. Commissural autologous pericardial patch repair: a novel technique for active mitral 
valve endocarditis involving the mitral annulus. Ann Thorac Surg. 2009;88: e29-30.

6. David TE. The use of pericardium in acquired heart disease: a review article. J Heart Valve Dis. 1998;7:13-8

7. Acar C, Saez de Ibarra J, Lansac E. Anterior leaflet augmentation with autologous pericardium for mitral repair in rheumatic mitral insufficiency. J Heart Valve Dis. 2004; 13:741-6.
8. Chauvaud S, Jebara V, Chachques J-C, El Asmar B, Mihaileanu S, Perier P, et al Valve extension with glutaraldehyde-preserved autologous pericardium: results in mitral valve repair. J Thorac Cardiovasc Surg. 1991;102:171-8.

9. Acar C, Tolan M, Berrebi A, Gaer J, Gouezo R, Marchix T, et al. Homograft replacement of the mitral valve: graft selection, technique of implantation, and results in forty-three patients. J Thorac Cardiovasc Surg. 1996;111: 367-80.

\title{
Open antegrade aortic stent implantation after surgical treatment in type A aortic dissection
}

\author{
Franz Masseli, MD, ${ }^{\mathrm{a}}$ Kai Wilhelm, MD, PhD,${ }^{\mathrm{b}}$ Chris Probst, MD, ${ }^{\mathrm{a}}$ and Wolfgang Schiller, MD, ${ }^{\mathrm{a}}$ Bonn, \\ Germany
}

Major complications after surgery for Stanford type A aortic dissections are aortic rupture and malperfusion of abdominal organs. The choice between subsequent surgical replacement of the descending aorta and retrograde stent placement via the femoral artery depends on the size of the true lumen and the location, as well as the extent of the intimal tear. Because the stent graft has to be advanced through segments with decreased wall strength, aortic rupture may be caused during the passage or the stent may be released in the false lumen. ${ }^{1}$ To minimize these risks, interventional techniques using an antegrade approach via the subclavian artery or the use of a hybrid procedure with open implantation of an aortic stent before placement of the arch prosthesis have recently been described. ${ }^{2-4} \mathrm{We}$ developed a new and safe technique of antegrade stent graft placement via the already implanted prosthesis either after resternotomy or during the original procedure. This technique aims to improve patient survival while minimizing postoperative complications because the stent is inserted through the firmest aortic segment, the prosthesis, in an antegrade fashion.

\section{MATERIALS AND METHODS}

The stent graft placement depends on the extent of the previously implanted aortic prosthesis and, in cases of concomitant arch replacement, on the technique used for the connection of the supraaortic vessels to the prosthesis (Figure 1). The preoperative imaging method of choice for this evaluation is an electrocardiogram-gated, multislice computed tomography (CT) examination of the entire aorta and supra-aortic branches.

From the Departments of Cardiac Surgery ${ }^{\mathrm{a}}$ and Radiology, ${ }^{\mathrm{b}}$ University Hospital Bonn, Bonn, Germany.

Disclosures: Authors have nothing to disclose with regard to commercial support. Received for publication May 7, 2012; revisions received June 30, 2012; accepted for publication July 30, 2012; available ahead of print Aug 30, 2012.

Address for reprints: Franz Masseli, MD, Sigmund-Freud St 25, 53127 Bonn, Germany (E-mail: fmasseli@uni-bonn.de).

J Thorac Cardiovasc Surg 2012;144:1527-30

$0022-5223 / \$ 36.00$

Copyright $\odot 2012$ by The American Association for Thoracic Surgery

http://dx.doi.org/10.1016/j.jtcvs.2012.07.088
Before the procedure, written consent was obtained from the patient or the next of kin. The procedure was carried out on a Compassionate Use basis according to German law.

After sternotomy, surgical clips at the aortic wall or the implanted prosthesis can be used to mark the proximal landing zone of the stent. To allow an unimpeded delivery of the stent past the end of the introducer, the point of introduction (POI) is chosen at least $3 \mathrm{~cm}$ proximal to the desired landing zone. A purse-string suture is then placed around the POI at the wall of the prosthesis.

Heparin is administered, followed by the antegrade introduction of an angio/pigtail catheter into the true aortic lumen via a $7 \mathrm{~F}$ introducer sheath at the selected POI. The catheter is advanced into the aortic arch, and digital subtraction angiography is performed during breathhold to outline the aorta and supra-aortic branches and to identify the placed markers. The catheter is then exchanged for a flexible guidewire (Radifocus; Terumo Medical Corp, Eschborn, Germany). The introduction into the true lumen is verified using transesophageal echocardiography visualization. The flexible guidewire is then exchanged for a stiff guidewire (Lunderquist extra-stiff; Cook Medical Inc, Bloomington, Ind). A stent graft with a suitable design for antegrade delivery is selected, and its introducer sheet is marked approximately $3 \mathrm{~cm}$ away from its end, depending on the distance of the POI to the planned proximal landing zone. After removing the catheter and the $7 F$ sheath, the introducer is advanced over the guidewire up to the $3-\mathrm{cm}$ mark. A small incision while passing the wall of the prosthesis is performed to facilitate the insertion. Under radiographic control, the stent is advanced via the introducer. In cases in which the POI is located proximal to the arch, the stent graft is manually bent before insertion to facilitate an easy passage through the aortic arch. The insertion is continued up to the desired position, which is verified by fluoroscopy via its projection in relation to the radio-opaque clips. The stent is then released, and the introducer system is removed.

During the release of the stent graft, blood pressure is decreased to less than $100 \mathrm{~mm} \mathrm{Hg}$ systolic; because of the fast-expanding capacity, no further modulation of cardiac output, such as rapid pacing, is necessary. The proximal aspect of the stent can be secured in the region of the overlapping landing zone with 2 Teflon-armed transmural sutures to prevent dislocation of the device. After radiographic control of accurate stent placement, the guidewire and introducer sheet are removed while simultaneously tightening the purse-string sutures. In cases with prior arch replacement, the proximal landing zone should be within the distal portion of the implanted prosthesis.

\section{RESULTS}

To the present, we have used this technique in a series of 5 patients with type A aortic dissection. None of the patients 